\title{
Redshift, metallicity and size of two extended dwarf Irregular galaxies. A link between dwarf Irregulars and ultra diffuse galaxies?^
}

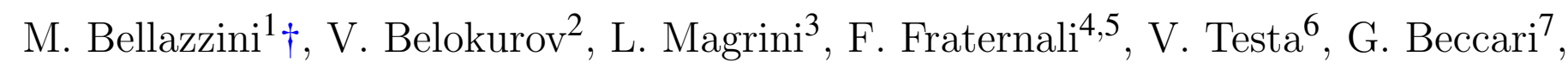
A. Marchetti ${ }^{8,9}$, R. Carini 6

${ }^{1}$ INAF - Osservatorio Astronomico di Bologna, Via Ranzani 1, 40127 Bologna, Italy

${ }^{2}$ Institute of Astronomy, University of Cambridge, Madingley Rd, Cambridge CB3 OHA, UK

${ }^{3}$ INAF - Osservatorio Astrofisico di Arcetri, Largo E. Fermi 5, 50125 Firenze, Italy

${ }^{4}$ Dipartimento di Fisica 63 Astronomia, Università degli Studi di Bologna, Viale Berti Pichat, 6/2, I - 40127 Bologna, Italy

${ }^{5}$ Leiden Observatory, Leiden University, Postbus 9513, 2300 RA, Leiden, The Netherlands

${ }^{6}$ INAF - Osservatorio Astronomico di Roma, via Frascati 33, 00040 Monteporzio, Italy

${ }^{7}$ European Southern Observatory, Karl-Schwarzschild-Strasse 2, 85748 Garching bei München, Germany

${ }^{8}$ Università degli Studi di Milano, via G. Celoria 16, 20130 Milano, Italy

${ }^{9}$ INAF - Osservatorio Astronomico di Brera, via Brera 28, 20122 Milano, via E. Bianchi 46, 23807 Merate, Italy

Accepted XXX. Received YYY; in original form ZZZ

\begin{abstract}
We present the results of the spectroscopic and photometric follow-up of two field galaxies that were selected as possible stellar counterparts of local high velocity clouds. Our analysis shows that the two systems are distant (D>20 Mpc) dwarf irregular galaxies unrelated to the local H I clouds. However, the newly derived distance and structural parameters reveal that the two galaxies have luminosities and effective radii very similar to the recently identified ultra diffuse galaxies (UDGs). At odds with classical UDGs, they are remarkably isolated, having no known giant galaxy within $\sim 2.0 \mathrm{Mpc}$. Moreover, one of them has a very high gas content compared to galaxies of similar stellar mass, with a $\mathrm{H}$ I to stellar mass ratio $M_{\mathrm{HI}} / M_{\star} \sim 90$, typical of almostdark dwarfs. Expanding on this finding, we show that extended dwarf irregulars overlap the distribution of UDGs in the $M_{V}$ vs. $\log r_{\mathrm{e}}$ plane and that the sequence including dwarf spheroidals, dwarf irregulars and UDGs appears as continuously populated in this plane. This may suggest an evolutionary link between dwarf irregulars and UDGs.
\end{abstract}

Key words: ISM: HiI regions — galaxies: dwarf — galaxies: star formation

\section{INTRODUCTION}

The complete census of dwarf galaxies in the Local Group (LG, and in the Local Volume) is a key observational enterprise in these decades, closely tied to the solution of the

\footnotetext{
* Based on data acquired using the Large Binocular Telescope (LBT). The LBT is an international collaboration among institutions in the United States, Italy, and Germany. LBT Corporation partners are The University of Arizona on behalf of the Arizona university system; Istituto Nazionale di Astrofisica, Italy; LBT Beteiligungsgesellschaft, Germany, representing the MaxPlanck Society, the Astrophysical Institute Potsdam, and Heidelberg University; The Ohio State University; and The Research Corporation, on behalf of The University of Notre Dame, University of Minnesota and University of Virginia.

$\dagger$ E-mail: michele.bellazzini@oabo.inaf.it
}

long-standing missing satellites problem (Moore et al. 1999; Sawala et al. 2016, and references therein). The recent discovery of the nearby $(\mathrm{D}=1.7 \mathrm{Mpc})$ faint $\left(M_{V}=-9.4\right)$ starforming dwarf galaxy Leo P (Giovanelli et al. 2013; McQuinn et al. 2013) has opened a new road for the identification of local dwarfs. Leo P was found as the stellar counterpart of a very compact high velocity cloud (CHVC) of neutral Hydrogen identified in the ALFALFA HI survey (Giovanelli et al. 2007), thus suggesting that some of the missing dwarfs in the LG and its surroundings could be hidden within similar CHVCs. These dwarfs may be the gas-rich star-forming counterparts of the quiescent ultra faint dwarfs (UFD) that have been found in relatively large numbers as stellar overdensities in panoramic imaging surveys (see, e.g., Belokurov 2013; Koposov et al. 2015, and references therein). Indeed there are models within the $\Lambda$-cold dark matter (CDM) sce- 
nario predicting that a large number of small DM haloes $\left(M \lesssim 10^{9} M_{\odot}\right.$, mini-haloes, after Ricotti 2009) should have had their star formation inhibited or quenched by global or local feedback effects (e.g., re-ionization, supernova feedback, ram-pressure stripping), thus leading to a population of gas-rich dwarfs with low or null stellar content (Ricotti 2009; Kormendy \& Freeman 2016).

The only possibility to confirm these systems as real galaxies and to gauge their distances is to find a stellar population associated with the HI clouds and indeed several teams followed up the CHVCs proposed by the ALFALFA (Adams et al. 2013) and GALFA-HI (Saul et al. 2012) surveys as candidate local $(\mathrm{D} \leq 3.0 \mathrm{Mpc})$ mini-haloes. Bellazzini et al. (2015a, B15a hereafter), within the SECCO survey $^{1}$, obtained deep and homogeneous imaging of 25 of the ALFALFA candidates from A13, finding only one confirmed stellar counterpart, the very faint star-forming system SECCO 1, likely located in the Virgo cluster (Bellazzini et al. 2015b). Sand et al. (2015), searching several public image archives, were able to confirm SECCO 1 and discovered four additional counterparts in the GALFA-HI sample, all of them with $\mathrm{D} \gtrsim 3.0 \mathrm{Mpc}$ (see also Tollerud et al. 2016). James et al. (2015, J15 hereafter) adopted a different approach, searching for small groupings of blue stars within the SDSS catalogue and identifying $\sim 100$ interesting candidates. The follow-up of 12 of them revealed a population of faint, blue, metal-poor low surface brightness (LSB) dwarfs in the distance range $5 \mathrm{Mpc} \lesssim \mathrm{D} \lesssim 120 \mathrm{Mpc}$, six of them associated with HI clouds listed in Giovanelli et al. (2007). J15 defined the newly found systems as blue diffuse dwarf (BDD) galaxies. Apparently we are beginning to scratch the surface of a population of LSB star-forming dwarfs that went undetected until now, although they are not found in the Local Volume (Sand et al. 2015; Beccari et al. 2016).

Within this context, we have selected mini-halo candidates from the GASS HI survey (McClure-Griffiths et al. 2009). Unlike previous searches, which only looked at velocities very different from Galactic emission (HVCs with $\left|v_{\mathrm{dev}}\right|>90 \mathrm{~km} \mathrm{~s}^{-1}, v_{\mathrm{dev}}$ being the deviation velocity with respect to a regularly rotating Galactic disc), we have explored the range of lower velocities, typical of intermediate velocity clouds (IVCs, $30<\left|v_{\text {dev }}\right|<90 \mathrm{~km} \mathrm{~s}^{-1}$ ). We detected HI sources using the code ${ }^{3 \mathrm{D}}$ BAROLO (Di Teodoro \& Fraternali 2015) and applying selection criteria on their size and velocity width to minimize the contamination from Galactic clouds. This left us with a sample of about one thousand best candidates, presumably with a very high degree of contamination by Galactic sources, which we searched for stellar counterparts in SDSS (Ahn et al. 2012), ATLAS (Shanks et al. 2015) and DES (Abbott et al. 2005) images. The process of visual inspection of available images around the positions of the clouds led to the selection of two promising candidates. These are blue LSB galaxies whose apparent diameters are fully compatible with being located within $\sim 3.0 \mathrm{Mpc}$ from us; moreover they are not completely unresolved, displaying a few blue compact sources resembling HII regions. Unfortunately, the spatial resolution of GASS is about 16 arcmin and it does not allow an association with certainty, hence spectroscopic follow-up is required.
Here we present the results of this follow-up, ultimately resulting in the rejection of the association of both the candidate stellar counterparts with the local gas clouds, since they are located at distances larger than $40 \mathrm{Mpc}$. Still, our observations provide the first redshift and metallicity estimates for these galaxies, which are useful for future studies, and reveal their remarkably large size, given their total luminosity. The latter feature lead us to note that the most luminous dwarf Irregular galaxies (dIrr) display structural parameters (sizes, integrated magnitudes, Sérsic indices, and surface brightnesses) overlapping the range inhabited by the newly discovered ultra diffuse galaxies (UDGs; van Dokkum et al. 2015a; Koda et al. 2015), suggesting a possible relation between the two classes of stellar systems.

While these observations are not part of the SECCO survey, they are strongly related and for this reason we adopt the SECCO nomenclature to name the two dwarfs considered here. In particular, following Beccari et al. (2016) we call them SECCO-dI-1 and SECCO-dI-2 (where dI = dwarf Irregular), abbreviated as SdI-1 and SdI-2.

\section{OBSERVATIONS AND DATA REDUCTION}

All the observations have been obtained under clear sky, during the night of March 3, 2016 with the Large Binocular Telescope (LBT) on Mt Graham (AZ), used in pseudobinocular mode, i.e. with different instruments operating simultaneously in the two channels of the telescope.

The main programme was performed using the low resolution spectrograph MODS-1 (Pogge et al. 2010), aligning a $1.2^{\prime \prime}$-wide long-slit along the major axes of the two targets. With this set-up and a dichroic, MODS-1 provides a blue spectrum covering $3200<\lambda<5650$, and a red spectrum covering $5650<\lambda<10000$ at a spectral resolution $\frac{\lambda}{\Delta \lambda} \sim 1100$. Three $t_{\exp }=1200 \mathrm{~s}$ exposures per target were acquired. SDSS images were used to define the slit position and orientation. The spectra were corrected for bias and flat-field, skysubtracted, wavelength calibrated, then extracted and combined into flux-calibrated summed spectra using the pipeline developed at the Italian LBT Spectroscopic Reduction Cen$t e r^{2}$.

Simultaneously, we got deep $\mathrm{r}_{\text {SDSS }}$ and isDSS (hereafter $\mathrm{r}$ and $\mathrm{i}$, for brevity) band imaging with LBC-R, the red channel of a pair of twin wide field $\left(\simeq 23^{\prime} \times 23^{\prime}\right)$ cameras (Giallongo et al. 2008). For each target, we got $9 \times 200 \mathrm{~s}$ exposures per filter, dithered to minimise the effect of bad pixels and to cover the inter-chip gaps of LBC-R. The reduction of the LBC images was performed with the specific pipeline developed at INAF-OAR (Paris et al. in preparation). The individual raw images were first corrected for bias and flat-field, and then background-subtracted. After astrometric calibration, they were combined into single $r$ - and i-band stacked images with the SWarp software (Bertin et al. 2002). In the following we will analyse these stacked and sky-subtracted images. The 5- $\sigma$ level over the background measured on the images corresponds to an i-band surface brightness of $\simeq 27.8 \mathrm{mag} / \operatorname{arcsec}^{2}$, in line with the limits typically obtained with LBC images reduced in the same way 


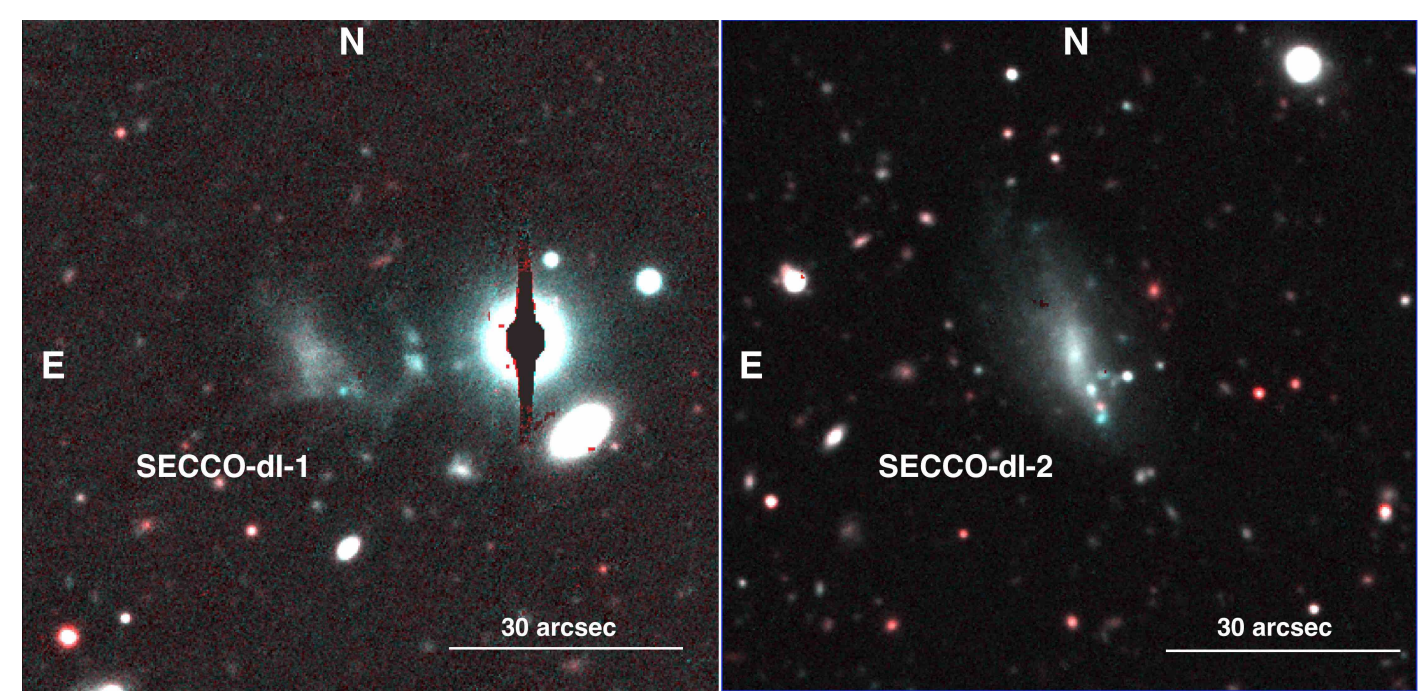

Figure 1. RGB color images of SECCO-dI-1 (left panel) and SECCO-dI-2 (right panel) obtained from our stacked LBC-RED images, using the $\mathrm{i}$ band for $\mathrm{R}$ and the $\mathrm{r}$ band for $\mathrm{G}$ and $\mathrm{B}$. The scale and the orientation are shown.

(see Beccari et al. 2016). The photometric calibration was obtained with hundreds of stars in common with the Sloan Digital Sky Survey - Data Release 9 (SDSS-DR9, Ahn et al. 2012). In Fig. 1 we show postage-stamp images of the two target galaxies from our LBC-R images.

\subsection{The targets}

The main properties of the target galaxies are summarised in Table 1. SdI-1 has a determination of redshift $z=0.025988$ from H I by Roberts et al. (2004) that places it far beyond the realm of local mini-haloes $(\mathrm{D} \leq 3 \mathrm{Mpc}$; Adams et al. 2013). However a negative redshift $(z=-0.05545)$ was also reported from optical spectroscopy (Liske et al. 2003) ${ }^{3}$. While extremely implausible, this may suggest an improper association between the $\mathrm{H}$ I detection by Roberts et al. (2004) and the stellar counterpart. Given the identification of an ICV along the same line of sight, a new attempt to get a reliable velocity from an optical spectrum was worth doing. Roberts et al. (2004) also reported an integrated magnitude of $\mathrm{B}=19.2$ and an effective radius $r_{\mathrm{e}}=4^{\prime \prime}$ (no uncertainty reported) from single-band photometry much shallower than the one we obtained with LBC.

SdI-2 lacks any redshift, size and optical magnitude estimate. There are two GALEX sources projected onto the main body of the galaxy, GALEXMSC J114433.79-005200.0 classified as a UV source, and GALEXASC J114433.60005203.0 classified as a galaxy.

Our deep images (see Fig. 1) reveal two remarkably elongated irregular blue galaxies, with some compact knots superimposed. SdI-1 may be interacting with some smaller companions, located $\sim 15^{\prime \prime}$ westward of its center. The main body of SdI-2 is surrounded by a very low SB asymmetric halo, more extended in the North-East direction. A fluffy nucleus is visible at the very center of SdI-2, which can be perceived also in the light profile (as a slight change of slope

\footnotetext{
3 In the Millennium Galaxy Catalog http://www.hs.unihamburg.de/jliske/mgc/
}

in the innermost $2^{\prime \prime}$, see below). While SdI-2 displays and an overall elliptical shape, the apparent morphology of SdI-1 is remarkably irregular independently of the adopted image cuts.

According to the NASA Extragalactic Datasystem $\mathrm{NED}^{4}$ there are eight galaxies within 1 degree of SdI1 with recessional velocities in the range $6000 \mathrm{~km} \mathrm{~s}^{-1} \leq$ $V_{r} \leq 10000 \mathrm{~km} \mathrm{~s}^{-1}$. The most remarkable one is the radio galaxy IC 753 that has $V_{r}=6220 \mathrm{~km} \mathrm{~s}^{-1}$ and lies $\simeq 1^{\circ}$ apart in the plane of the sky, corresponding to a projected distance of $\simeq 2.0 \mathrm{Mpc}$ at the distance of SdI-1. Two galaxies (SDSS J115406.35+000158.5 and SDSS J115254.30001408.5 ) have velocities within $200 \mathrm{~km} \mathrm{~s}^{-1}$ of SdI-1. They are relatively faint $(\mathrm{r} \geq 17.7)$ and lie at a projected distance of $0.9 \mathrm{Mpc}$ and $1.6 \mathrm{Mpc}$, respectively.

There are eighteen galaxies listed in NED within 1 degree of SdI-2 with recessional velocities in the range $1000.0 \mathrm{~km} \mathrm{~s} \mathrm{~s}^{-1} \leq V_{r} \leq 4000.0 \quad \mathrm{~km} \mathrm{~s}^{-1}$, but only three within $\simeq 500 \mathrm{~km} \mathrm{~s}^{-1}$ of SdI-2, SDSS J114428.88-012335.7, SDSS J114640.78-011749.2, and SDSS J114325.36-013742.5. All these three are significantly fainter than SdI-2 ( $r \geq 20.8)$ and lie more than half a degree apart, corresponding to a projected distance larger than $350 \mathrm{kpc}$ at the distance of SdI-2. In conclusion, both our target dwarfs do not lie in the vicinity of large galaxies and seems to be remarkably isolated.

\section{PHYSICAL PROPERTIES OF SDI-1 AND SDI-2}

In Fig. 2 we show relevant portions of the spectra we obtained from our observations. In SdI-1 this corresponds to a bright and extended H II region $\simeq 5.2^{\prime \prime}$ to the south-west of the center of the galaxy. In SdI-2 we got the spectra of two such $\mathrm{H}$ II regions (sources $\mathrm{A}$ and $\mathrm{B}, \simeq 5.3^{\prime \prime}$ and $\simeq 8.4^{\prime \prime}$ to the south-south-west of the center of the galaxy, respectively)

\footnotetext{
${ }^{4}$ http://ned.ipac.caltech.edu
} 
Table 1. Mean Properties of the targets

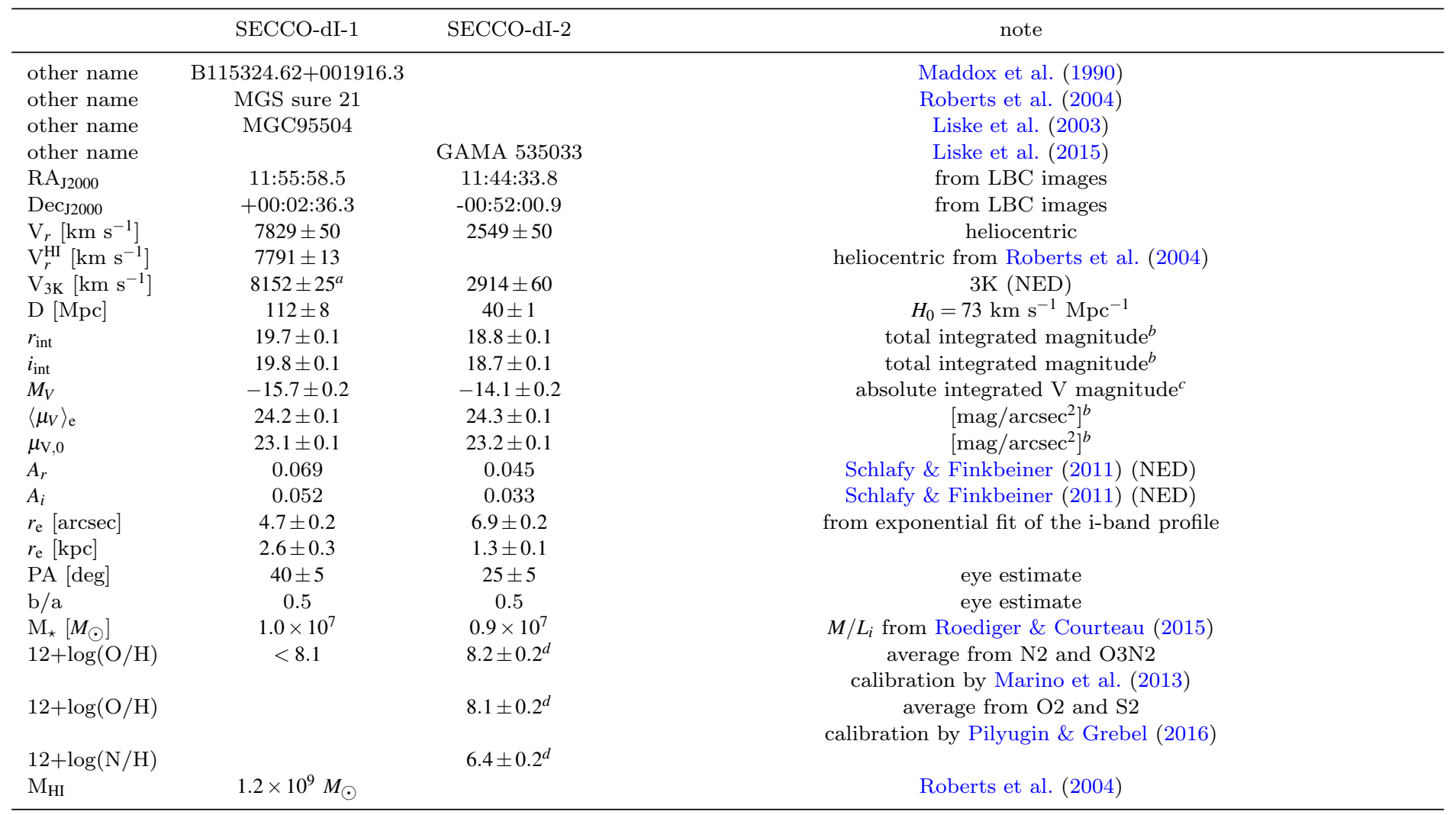

${ }^{a}$ Derived from the H I radial velocity estimate by Roberts et al. (2004)

${ }^{b}$ Not corrected for extinction.

${ }^{c}$ i- and $-\mathrm{r}$ band magnitudes have been transformed into $\mathrm{V}$ magnitudes, using the equation $\mathrm{V}=\mathrm{r}+0.813(\mathrm{r}-\mathrm{i})+0.050$, valid for $-0.3 \leq \mathrm{r}-\mathrm{i} \leq 0.7$, that we derived in the same way as Lupton (2005) from the set of standard stars in the globular cluster NGC 2419 by Stetson (2005).

${ }^{d}$ Weighted mean of the estimates for sources SdI-2 A and SdI-2 B.

and the absorption spectrum of the central nucleus (source C). To get a rough estimate of the mean age of the nucleus we compared the observed spectrum of source $\mathrm{C}$ with a set of low-resolution synthetic spectra of simple stellar populations from the BASTI repository (Percival et al. 2009; Pietrinferni et al. 2004), finding a satisfactory fit with a model having metallicity $\mathrm{Z}=0.002$ and age $=200 \mathrm{Myr}$.

We estimated the redshift by fitting the centroid of identified atomic lines and averaging the various measures. The uncertainty in the derived radial velocities is dominated by the uncertainty in the zero point which, comparing the velocities from the blue and red spectra, is about $50 \mathrm{~km} \mathrm{~s}^{-1}$. The match between our new optical redshift for SdI-1 and the H I estimate by Roberts et al. (2004) implies that the association between the distant cloud and the stellar counterpart is correct. Both SdI-1 and SdI-2 have velocities clearly incompatible with association with our GASS candidate minihaloes. Since these were the best candidates for counterparts selected from a sample of $\sim 1000 \mathrm{ICVs}$, we conclude that it is very unlikely that a local dwarf is associated with these clouds.

We report the radial velocities in the $3 \mathrm{~K}$ reference frame and then we derive the distance adopting $H_{0}=$ $73 \mathrm{~km} \mathrm{~s}^{-1} \mathrm{Mpc}^{-1}$ (the value adopted by NED). We obtain a distance of $112 \pm 8 \mathrm{Mpc}$ and $40 \pm 1 \mathrm{Mpc}$ for SdI-1 and SdI-2, respectively.

\subsection{Metallicity}

Using the IRAF task splot we measured the fluxes of recombination lines of $\mathrm{H}(\mathrm{H} \alpha$ and $\mathrm{H} \beta)$ and collisional lines of a few ions ([OII], [OIII], [NII], [SII], see Table 2). All measured line intensities were corrected for extinction by computing the ratio between the observed and theoretical Balmer decrement for the typical conditions of an Hil region (see Osterbrock \& Ferland 2006). For the metallicity estimates in SdI-2, described below, we adopt the weighted average of the estimates of the two individual H II regions, as their abundances are indistinguishable, within the uncertainties.

Due to the absence of electron-temperature diagnostic lines, the gas-phase oxygen abundance of each source was determined with the following strong-line ratios (also depending on the available lines in each spectrum $): \mathrm{N} 2=[\mathrm{NII}] / \mathrm{H} \alpha, \quad \mathrm{O} 3 \mathrm{~N} 2=([\mathrm{OIII}] / \mathrm{H} \beta) /([\mathrm{NII}] / \mathrm{H} \alpha)$, $\mathrm{O} 2=([\mathrm{OII} / \mathrm{H} \beta)$, and $\mathrm{S} 2=([\mathrm{SII}](\lambda 6717+\lambda 6730)) / \mathrm{H} \alpha$. For N2 and O3N2 we adopt the calibration by Marino et al. (2013), while for $\mathrm{O} 2$ and $\mathrm{S} 2$ we adopt the calibration by Pilyugin \& Grebel (2016). The final abundance estimates, listed in 

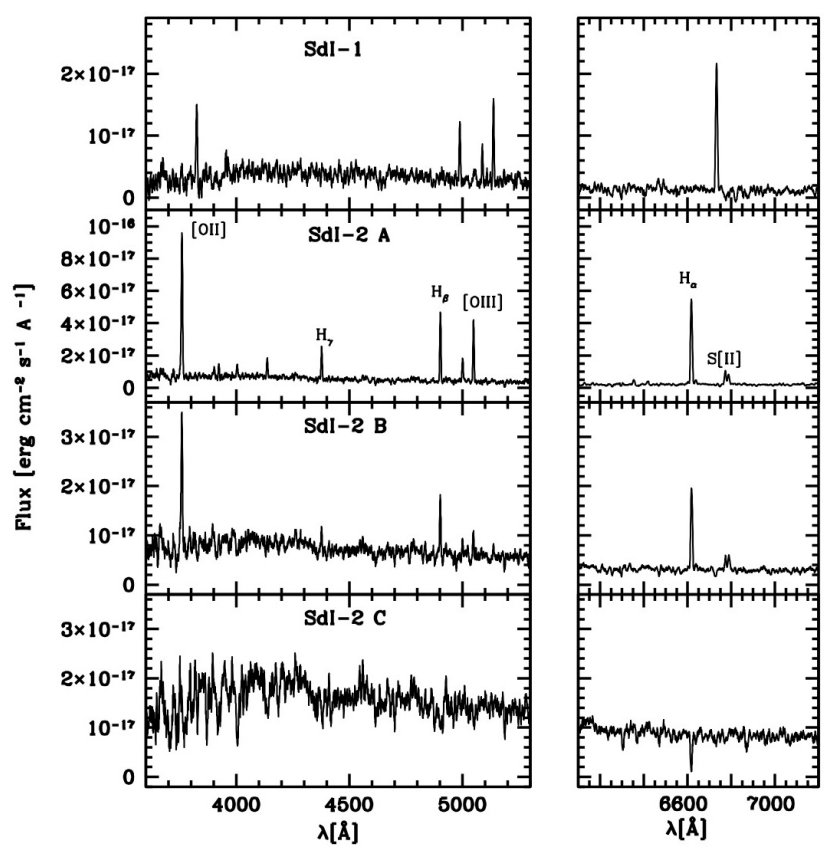

Figure 2. The most significant portions of the spectra from our long-slit observations of SdI1 and SdI2. Upper panel: the single $\mathrm{H}$ II region in SdI-1. Middle panels: the two H II regions identified in SdI-2. Lower panel: the stellar nucleus of SdI-2. Some noteworthy lines have been labelled in the spectrum of SdI-2 A.

Table 1, are the average of the values obtained from N2 and $\mathrm{O} 3 \mathrm{~N} 2$ and the average of the values obtained from $\mathrm{O} 2$ and S2. For SdI-2 the two estimates are in good agreement, within the uncertainties. For SdI-1 we were able to obtain only an upper limit from N2 and O3N2. For both galaxies, the derived abundances are within the range covered by dwarf galaxies of similar luminosity (see, e.g., Lee et al. 2003). The nitrogen-to-oxygen ratio is about $\log (\mathrm{N} / \mathrm{O}) \simeq$ -1.7 in SdI-2, indicating that the star formation efficiency is quite high in this galaxy $\left(\simeq 3-5 \mathrm{Gyr}^{-1}\right.$, Vincenzo et al. 2016).

\subsection{Surface Photometry}

In Fig. 3 we show the i-band surface brightness profiles of our target galaxies, obtained by photometry on elliptical apertures performed with the APT software (Laher et al. 2012). The axis ratio and position angle were estimated by eye, superposing ellipses to the images of the galaxies. r-band profiles were obtained in the same way and are very similar in shape, but we prefer to analyse i-band profiles because they should be minimally affected by the light of bright H II regions. Indeed slightly larger effective radii are obtained from r-band profiles.

In spite of some noise due to the presence of fore/background sources or compact sources within the galaxies, the overall profiles emerge very clearly, and in both cases they can be satisfactorily fitted by a simple exponential model. Contamination from nearby but unrelated sources especially affects the outer part of the profile of SdI-1, which flattens beyond $R=10^{\prime \prime}$ mainly due to the contribution from

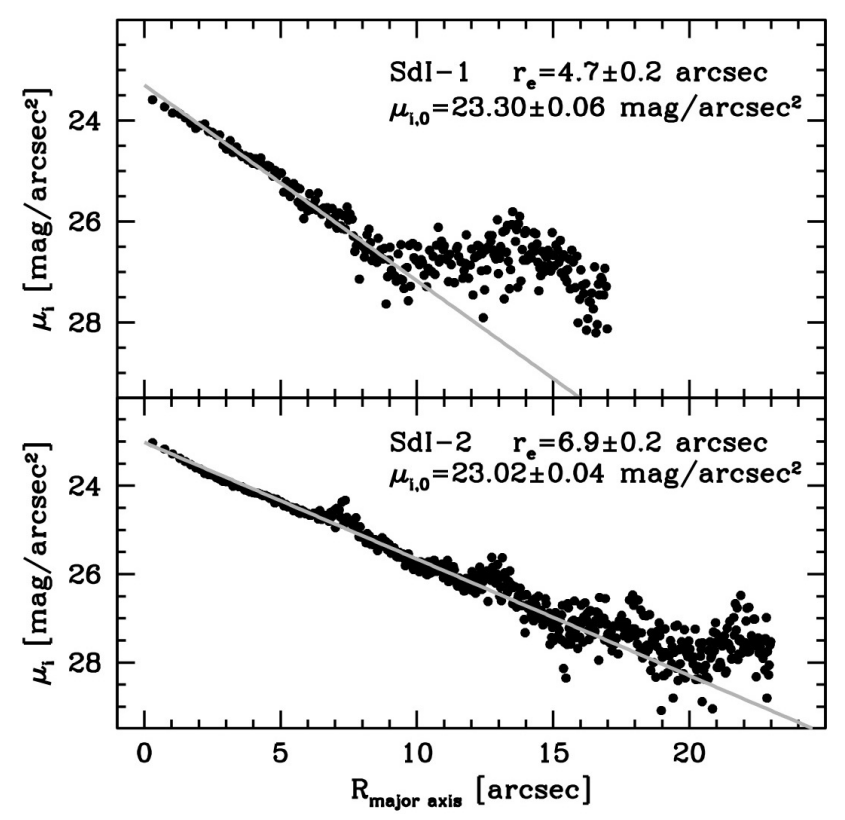

Figure 3. i-band surface brightness profiles of SdI-1 (upper panel) and SdI-2 (lower panel) from surface photometry on elliptical apertures (with b/a and PA as reported in Tab. 1. The grey lines are exponential profiles that best fit the observed profiles. The best-fit estimates of $r_{\mathrm{e}}$ and $\mu_{\mathrm{i}, 0}$ are also reported.

the possible companion lying to the west of the galaxy (see Sect. 2.1). We limited our fit to the nearly uncontaminated inner regions to avoid overestimating the size of the galaxies. The effective radii $\left(r_{\mathrm{e}}\right)$ along the major axis, the central surface brightness $\left(\mu_{\mathrm{V}, 0}\right)$ and the associated uncertainties have been estimated by performing a linear regression on the points within $R=10.0^{\prime \prime}$, for SdI- 1 , and within $R=16.0^{\prime \prime}$, for SdI-1, with the macro lm of the package $\mathrm{R}^{5}$. This simple approach seems fully adequate in this context, is straightforward and provides robust estimates of these parameters.

We note that our $r_{\mathrm{e}}$ value for SdI-1 is in reasonable agreement with the estimate by Roberts et al. (2004) from much shallower images. To minimise the effect of fore/background sources we derived the integrated magnitudes and the mean surface brightness within $r_{\mathrm{e}}$ from the best fitting exponential profiles, using the equations provided by Graham \& Driver (2005). All the photometric and structural parameters derived in this way are listed in Table 1 .

The $(\mathrm{r}-\mathrm{i})_{0}$ colors of SdI- 1 and SdI- $2(-0.1$ and +0.1 , respectively) are typical of star-forming galaxies and on the blue side of the range spanned by blue UDGs from Roman \& Trujillo (2016, see Sect. 4 for a comparison with these galaxies). Following these authors we used the relations by Roediger \& Courteau (2015) to derive a rough estimate of the stellar mass from the integrated i-band luminosity and the $(r-i)_{0}$ colors; both galaxies have $M_{\star} \simeq 1 \times 10^{7} M_{\odot}$ (see Tab. 1). For SdI-1 we have also a reliable estimate of the H I mass from Roberts et al. (2004) that allows us to compute the ratio of the H I mass to V-band luminosity (from Tab. 1)

5 www.r-project.org 
Table 2. Extinction-corrected fluxes of emission lines (assuming $\mathrm{H}_{\beta}$ flux=100.0)

\begin{tabular}{lccc}
\hline line & SECCO-dI-1 & SECCO-dI-2 A & SECCO-dI-2 B \\
\hline$[\mathrm{OII}]_{3726+3729}$ & $144.5 \pm 9.2$ & $256.2 \pm 15.5$ & $252.2 \pm 13.4$ \\
$\mathrm{H}_{\delta}$ & - & $25.7 \pm 2.1$ & - \\
$\mathrm{H}_{\gamma}$ & - & $46.8 \pm 3.1$ & - \\
$\mathrm{H}_{\beta}$ & $100.0 \pm 8.4$ & $100.0 \pm 7.8$ & $100.0 \pm 5.8$ \\
{$[\mathrm{OIII}]_{4959}$} & $46.7 \pm 5.7$ & $27.5 \pm 2.2$ & - \\
{$[\mathrm{OIII}]_{5007}$} & $119.5 \pm 9.5$ & $40.9 \pm 4.9$ & $82.4 \pm 4.9$ \\
$\mathrm{H}_{\alpha}$ & $289.1 \pm 21.7$ & $287.1 \pm 14.0$ & $287.0 \pm 11.4$ \\
{$[\mathrm{NII}]_{6584}$} & $<6.0$ & $10.6 \pm 3.2$ & $15.5 \pm 1.4$ \\
{$[\mathrm{SII}]_{6717}$} & - & $18.1 \pm 3.5$ & $41.8 \pm 2.3$ \\
{$[\mathrm{SII}]_{6731}$} & - & $38.3 \pm 4.3$ & $31.3 \pm 2.0$ \\
\hline$\left[10^{17} \mathrm{erg} \mathrm{cm}^{-2} \mathrm{~s}^{-1}\right]$ & $5.9 \pm 0.5$ & $24.5 \pm 1.4$ & $7.1 \pm 0.5$ \\
\hline
\end{tabular}

to be $\frac{M_{\mathrm{HI}}}{L_{V}} \simeq 6.2$, larger than the typical values of gas-rich dwarfs in the Local Group $\left(\frac{M_{\mathrm{HI}}}{L_{V}} \sim 2\right.$; McConnachie 2012). The ratio of $\mathrm{H}$ I mass to stellar mass, $\frac{M_{\mathrm{HI}}}{M_{\star}} \simeq 90$ places SdI-1 in the realm of almost-dark galaxies (see Cannon et al. 2015; Beccari et al. 2017).

\section{A LINK BETWEEN DWARF IRREGULARS AND UDGS?}

Having at our disposal newly derived structural parameters and distances of our target galaxies, we noted that they have absolute magnitude, surface brightness (see Table 1), and, in particular, physical sizes $\left(r_{\mathrm{e}}=2.6 \mathrm{kpc}\right.$ and $r_{\mathrm{e}}=1.3 \mathrm{kpc}$, for SdI-1 and SdI-2, respectively) in the range covered by the recently identified class of ultra diffuse galaxies ${ }^{6}$ (UDG, van Dokkum et al. 2015a).

UDGs are roundish amorphous galaxies having "...the sizes of giants but the luminosity of dwarfs..." (Beasley et al. 2016) that have been recently discovered in large numbers in clusters of galaxies (see van Dokkum et al. 2015a; Koda et al. 2015; Mihos et al. 2015; Muñoz et al. 2015; van der Burg 2016, and references therein), with some examples in other environments (see Martínez-Delgado 2016; Yagi et al. 2016, and references and discussion therein). In general UDGs lie on the red sequence of galaxies and their light profiles are well approximated by exponential laws (Koda et al. 2015; Yagi et al. 2016). The fact that they survive in dense environments without obvious signs of tidal distortions, as well as the first analyses of the kinematics of individual UDGs, strongly suggest that they are dark matter dominated systems (van Dokkum et al. 2015a; van der Burg 2016; van Dokkum et al. 2016; Beasley et al. 2016; Zaritsky 2016). On

\footnotetext{
6 There is not yet a generally accepted definition of the class, hence similarity must be intended in a broad sense. For example, SdI-1 fulfills the main size criterion $\left(r_{\mathrm{e}}>1.5 \mathrm{kpc}\right)$ adopted by Yagi et al. (2016) in their definition of UDGs, independently of adopting the major axis or the circularised effective radius, SdI-2 fails by a small amount, and both galaxies fail to fulfill the $\mu_{\mathrm{V}, 0}>$ $24.0 \mathrm{mag} / \operatorname{arcsec}^{2}$ criterion. However both galaxies are consistent with all the Yagi et al. (2016) criteria once the fading by passive evolution is taken into account (see below). Moreover, both SdI-1 and SdI-2 overlap with Fornax UDGs identified by Muñoz et al. (2015) in luminosity, radius and $\langle\mu\rangle_{\mathrm{e}}$.
}

the other hand it is still not established if, e.g., they are failed giant galaxies (van Dokkum et al. 2015b) or extended and quenched dwarfs (Beasley \& Trujillo 2016).

In Fig. 4 we compare the sample of Coma cluster UDGs by van Dokkum et al. (2015a) with SdI-1, SdI-2 and the dwarf galaxies in the Local Volume, from the compilation by McConnachie (2012), in the absolute magnitude vs. effective radius plane. Local dIrr galaxies are plotted in a darker tone of grey with respect to dSphs and dwarf ellipticals. NGC 300 and NGC 55 have been plotted for reference although they do not fit the definition of dwarf galaxies. To expand our view we included also the BDDs of James et al. $(2015,2016)$ and the H I-selected sample of gas-rich LSB galaxies within $\simeq 250 \mathrm{Mpc}$ by Du et al. (2015). These authors derived new, more reliable estimates of integrated magnitudes and effective radii by re-analysing SDSS images. Absolute g- and r-band integrated magnitudes from Du et al. (2015) were transformed into V-band magnitudes by using Lupton $(2005)^{7}$ equations. Also g-band magnitudes of van Dokkum et al. (2015a) galaxies were converted into $\mathrm{V}$-band with the same equation, adopting the mean color of red sequence galaxies at that luminosity, $(\mathrm{g}-\mathrm{r})_{0}=0.6$, from Blanton \& Moustakas (2009). UDGs from Roman \& Trujillo (2016, see discussion below) have been included in Fig. 4 with the same transformation, adopting mean colors of $(\mathrm{g}-\mathrm{r})_{0}=0.25$ and $(\mathrm{g}-\mathrm{r})_{0}=0.6$, for blue and red UDGs, respectively (see their Fig. 4). In Fig. 4 we show two slightly different versions of the plot. In the lower panel we use the effective radius measured along the major axis of the galaxies $\left(r_{\mathrm{e}, \mathrm{maj}}\right)$, while in the upper panel we use the circularised radius $\left(r_{\mathrm{e}, \mathrm{circ}}=r_{\mathrm{e} \text {,maj }} \sqrt{b / a}\right)$, since this has been adopted in several comparisons of the same kind including UDGs (see, e.g., Beasley et al. 2016; van Dokkum et al. 2015b).

We note that, independently of the version of the plot, not only SdI-1 and SdI-2, but also a few local large dwarf irregulars partly overlap the distribution of UDGs (WLM, IC 1613, in particular, but also NGC 3109 and IC 3104, if $r_{\mathrm{e} \text {,maj }}$ is considered). Indeed, in their extensive literature search for previously identified UDGs, Yagi et al. (2016) found six galaxies satisfying all their UDG criteria in the catalog of nearby dwarf irregulars by Hunter \& Elmegreen

${ }^{7}$ http://www.sdss3.org/dr9/algorithms/sdssUBVRITransform.php 


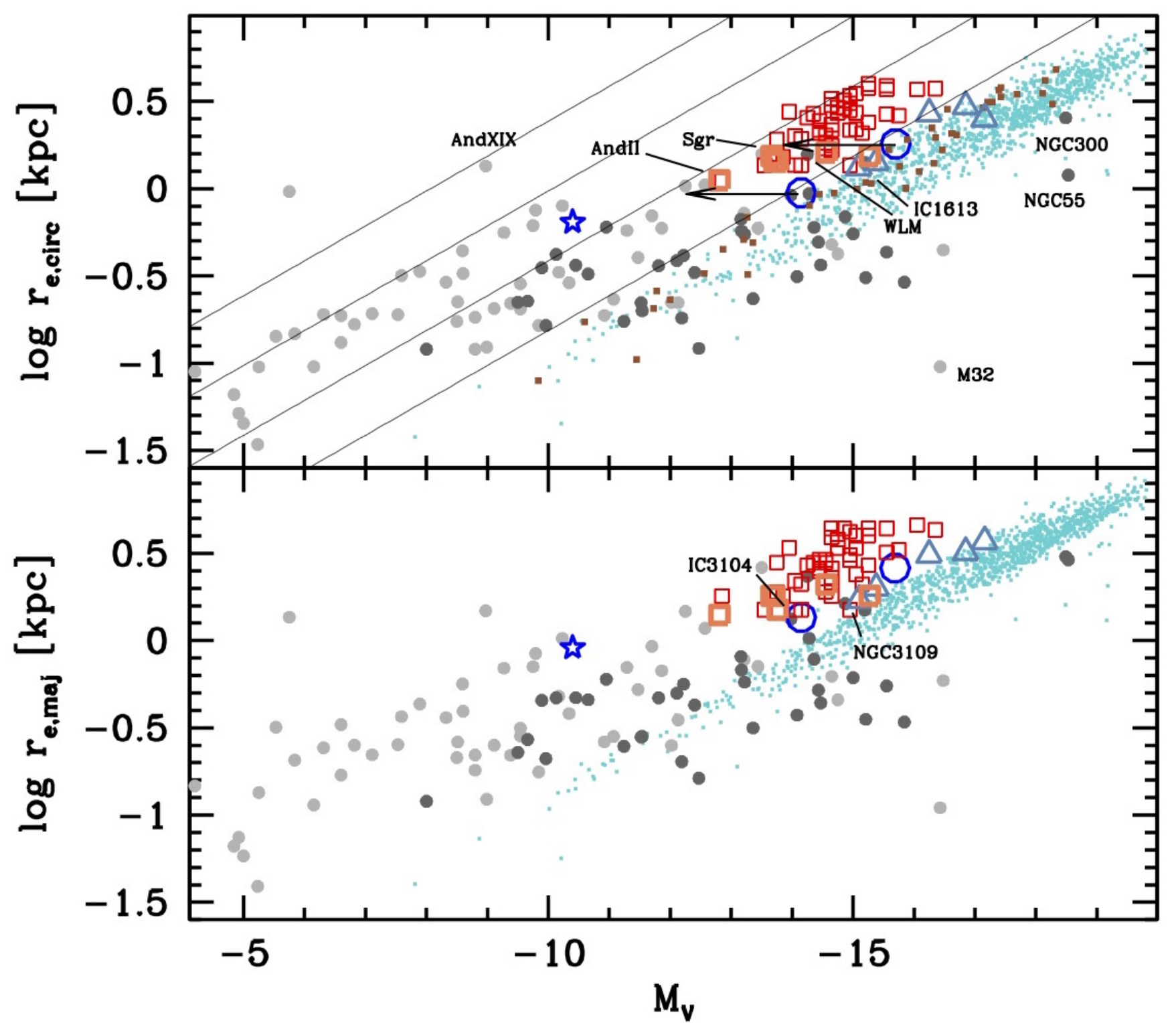

Figure 4. Comparison between SdI-1 and SdI-2 (open blue circles), local dwarf galaxies from the most recent version of the catalog by McConnachie (2012, grey filled circles; darker points are gas-rich galaxies, having $M_{\mathrm{HI}} / L_{V}>0.1$, in solar units), and UDGs from van Dokkum et al. (2015a, red empty squares) in the $M_{V}$ vs. effective radius plane. The arrows show the fading of a metal-poor single-burst stellar system (with the size and luminosity of SdI-1 and SdI-2) passively evolving from age $=0.5$ Gyr to age $=6.0$ Gyr, according to BASTI evolutionary models (Percival et al. 2009). Thick orange open squares are red UDGs and thick azure open triangles are blue UDGs from Roman \& Trujillo (2016). Turquoise points are gas-rich low SB galaxies from Du et al. (2015). Small brown filled squares are blue diffuse dwarfs (BDDs) from James et al. (2016, not plotted in the lower panel since only $r_{\mathrm{e}, \text { circ }}$ values were available). The blue open star is the almost-dark star forming galaxy SECCO 1, from Bellazzini et al. (2015b) and Beccari et al. (2017). The point labelled as Sgr is the Sagittarius dwarf spheroidal galaxy. In the lower panel the effective radius measured along the major axis is adopted $\left(r_{\mathrm{e}, \text { maj }}\right)$ while in the upper panel the circularised effective radius $\left(r_{\mathrm{e}, \mathrm{circ}}=r_{\mathrm{e}, \text { maj }} \sqrt{b / a}\right)$ is used. Some noteworthy galaxies have been labelled, for reference. In the upper panel, four $\left\langle\mu_{V}\right\rangle_{\mathrm{e}}=$ const. lines are also plotted, for reference, at $\left\langle\mu_{V}\right\rangle_{\mathrm{e}}=30.5,28.5,26.5,24.5 \mathrm{mag} / \mathrm{arcsec}^{2}$, from top to bottom.

(2006), concluding that an overlap between the two classes may indeed exist.

The adoption of $r_{\mathrm{e}, \mathrm{circ}}$ makes the overlapping range slightly narrower, due to the fact that UDGs have, on average, a much rounder shape than dIrrs. It is also important to stress that while it was generally recognised that in the $M_{V}$ vs. $\log r_{\mathrm{e}}$ plane, UDGs lie at the tip of the sequence of dSphs and dIrrs, the inclusion of the latter class of galaxies in the plot (usually not performed) makes the overall distribution much more continuous: there seems to be no gap between dSphs+dIrrs and UDGs. Expanding the view to non-local systems suggests that large dIrrs have indeed effective radii in the range 1-4 kpc (see, e.g., Lange et al. 2016, their Fig. 9, in particular).

The sharp edge of the distribution of Du et al. (2015) galaxies toward low SB values in Fig. 4 strongly suggests 
that the lack of a more substantial overlap with the UDGs may be merely due to incompleteness: a population of starforming dwarfs with $\left\langle\mu_{V}\right\rangle_{\mathrm{e}}>24.5 \mathrm{mag} / \operatorname{arcsec}^{2}$ may still be waiting to be uncovered by future more sensitive surveys ${ }^{8}$. This hypothesis is confirmed by the fact that the sample of BDDs by James et al. (2016), which has been selected from the same source (SDSS images), shows the same cut in surface brightness. Fig. 4 shows also that Du et al. (2015) and James et al. (2016) galaxies and local dIrrs are indistinguishable in this plane.

We do not know if the similarity between large dIrrs and $\mathrm{UDGs}^{9}$ is hinting at an evolutionary link between the two classes, but it is certainly worth noting, since a clear explanation for the origin of UDGs is still lacking and they can also be the end-product of different evolutionary channels (Zaritsky 2016). For instance, it seems to support the hypothesis that UDGs may be "quenched Large Magellanic Cloud-like systems", recently put forward by Beasley \& Trujillo (2016). It may be conceived that the tidal stirring process that is supposed to transform small gas-rich disc dwarfs into dSph around Milky Way-sized galaxies (Mayer et al. 2007), acting on a larger scale, can also transform large dIrrs into UDGs within galaxy clusters, removing the gas, stopping the star formation and redistributing the stars into an amorphous spheroid (see also Amorisco \& Loeb 2016 for a possible relation between disc galaxies and UDGs, and Burkert 2016 for shape arguments not supporting this relation). Interestingly, the only Local Group quiescent galaxy overlapping the distribution of UDGs in Fig. 4 (see also Yagi et al. 2016), the Sagittarius dSph, is believed to have evolved to its present amorphous and gas-less state from a star-forming galaxy of mass similar to the Small Magellanic Cloud, mainly driven by the tidal interaction with the Milky Way that is disrupting it (Majewski et al. 2003; Niederste-Ostholt et al. 2010). However, it must be noted, in this context, that typical UDGs do not shows signs of ongoing disruption. In any case, the continuity of the sequence including dIrrs of any size and dwarf spheroids of any size (dSphs and UDGs) in the $M_{V}$ vs. $\log r_{\mathrm{e}}$ plane may suggest similar progenitors for all these LSB systems over more than six orders of magnitude in luminosity.

In this context, the very recent work by Roman \& Trujillo (2016, RT16 hereafter) is particularly relevant. These authors identified a population of possible progenitors of UDGs (blue UDGs, from their colors significantly bluer than classical red UDGs) in the outskirts of galaxy groups containing classical UDGs. RT16 demonstrated that a few Gyrs of passive evolution would transform their blue UDGs into classical red ones, by reddening their colors, fading their surface brightness and total luminosity while keeping their large size nearly unchanged. SdI-1 and SdI-2 have size, stel-

\footnotetext{
${ }^{8}$ It is interesting to note that Yagi et al. (2016) stated that the data-reduction strategy they adopted implies a bias against the detection of extended dwarf irregulars.

9 The similarities include also the typical shape of the light profiles (nearly exponential), the incidence of stellar nuclei (Yagi et al. 2016), and the presence of globular cluster systems (Beasley et al. 2016; Beasley \& Trujillo 2016; van Dokkum et al. 2016). Note that at least one UDG has been found hosting a globular cluster population significantly larger than typical dwarfs of the same luminosity (van Dokkum et al. 2016).
}

lar mass, luminosity, surface brightness, ellipticity and color very similar to the RT16 blue UDGs (see, e.g., Fig. 4), hence RT16 results on the evolutionary path of blue UDGs applies also to our galaxies as well as to other local dIrrs plotted in Fig. 4. In particular, according to canonical solarscaled BASTI ${ }^{10}$ stellar evolutionary models (Percival et al. 2009; Pietrinferni et al. 2004) for a simple stellar population with metallicity $\mathrm{Z}=0.002$, the passive evolution from an age $=0.5$ Gyr to age $=6.0$ Gyr would led to a fading by 1.84 magnitudes in V-band, driving the central surface brightness of SdI-1 and SdI-2 down to $\mu_{\mathrm{V}, 0} \simeq 25.0 \mathrm{mag} / \mathrm{arcsec}^{2}$, fully in the realm of classical UDGs, as displayed by the arrows plotted in the upper panel of Fig. 4.

SdI-1 and SdI-2 seem to be even more isolated than their RT16 siblings, since they lie at more than $900 \mathrm{kpc}$ and $350 \mathrm{kpc}$ from their nearest known neighbours, respectively, while RT16 blue UDGs are within $250-550 \mathrm{kpc}$ from the centre of the galaxy groups they are associated to. Moreover their nearest neighbours are dwarf galaxies, while the groups where RT16 UDGs live host also giant galaxies. $S d I-1$ and SdI-2 are perhaps the most isolated UDGs (or UDG progenitors) identified until now, indicating that isolated field dwarfs can indeed evolve into UDGs. Hence, SdI-1 and SdI2 lend additional and independent support to the scenario proposed by Roman \& Trujillo (2016), in which the progenitors of classical UDGs were dwarfs born in the field and then processed within galaxy groups and, finally, in galaxy clusters.

The properties of SdI-1 and SdI-2 also fit nicely with those of the dwarfs identified by Di Cintio et al. (2017) as counterparts of UDGs in cosmological simulations including feedback processes. Also in the Di Cintio et al. (2017) scenario UDGs are born as dwarf galaxies, but their evolution is driven by internal processes (feedback-driven gas flows, in particular), hence it is independent of the environment and it is expected to take place also in the field. A specific prediction of the simulations by Di Cintio et al. (2017) is that UDGs evolved in isolation should have larger gas content than regular dwarfs of similar stellar mass. It is intriguing to note that this prediction is vindicated by the the very high $\mathrm{H} \mathrm{I} / M_{*}$ ratio observed in SdI-1, the most extended among our two isolated, star-forming UDGs.

\section{ACKNOWLEDGEMENTS}

We are grateful to an anonymous referee for a very careful reading of the original manuscript and for useful suggestions that allowed us to make a more thorough analysis. This research has made use of the NASA/IPAC Extragalactic Database (NED) which is operated by the Jet Propulsion Laboratory, California Institute of Technology, under contract with NASA. This research has made use of the SIMBAD database, operated at CDS, Strasbourg, France.

\section{REFERENCES}

Abbott, T., Aldering, G., Annis, J., et al., arXiv:astro$\mathrm{ph} / 0510346$

10 http://basti.oa-teramo.inaf.it 
Adams, E.K., Giovanelli, R., Haynes, M.P., 2013, ApJ, 768, 77 (A13)

Ahn, C.P., Alexandroff, R., Allende Prieto, C., et al. 2012, ApJS, 203, 21

Amorisco, N. C., Loeb, A., 2016, MNRAS, 459, L51

Beasley, M.A., Romanowsky, A., Pota, V., Navarro, I.M., Martinez Delgado, D., Neyer, F., Deich, A.L., 2016, ApJ, 819, L20

Beasley, M.A., Trujillo, I., 2016, ApJ, 830, 23

Beccari, G., Bellazzini, M., Battaglia, G., et al., 2016, A\&A, 591, 56 (B16)

Beccari, G., Bellazzini, M., Magrini, L., et al., 2017, MNRAS, 465, 2189

Bellazzini, M., Beccari, G., Battaglia, et al., 2015a, A\&A, 575, 126 (B15a)

Bellazzini, M., Magrini, L., Mucciarelli, A., et al., 2015b, ApJ, 800, L15 (B15b)

Belokurov, V., 2013, New Astronomy Rev., 57, 100

Bertin, E., Mellier, Y., Radovich, M., Missonnier, G., Didelon, P., Morin, B., 2002 in Astronomical Data Analysis Software and Systems XI, San Francisco: Astronomical Society of the Pacific, ASP Conf. Series, 281, 228

Blanton, M.R., Moustakas, J., 2009, ARA\&A, 47, 159

Burkert, A., 2016, ApJ, submitted (arXiv:1609.00052)

Cannon, J.M., et al., 2015, AJ, 149, 72

Di Cintio, A., Brook, C.B., Dutton, A.A., Macciò, A.V., Obreja, A., Dekel, A., 2017, MNRAS, 466, L1

Di Teodoro, E., Fraternali, F., 2015, MNRAS, 451, 3021

Du, W., Wu, H., Lam, M.I., Zhu, Y., Lei, F., Zhou, Z., 2015, AJ, 2015, 149, 199

Freudling, W., Romaniello, M., Bramich, D. M., et al. 2013, A\&A, 559, A96

Giallongo, E., Ragazzoni, R., Grazian, A., 2008, A\&A482, 349

Giovanelli, R., Haynes, M.P., Adams, E., 2013, AJ, 146, 15

Giovanelli, R., Haynes, M.P., Kent, B.R., et al., 2007, AJ, 133, 2583

Graham, A.W., Driver, S.P., 2005, Publ. Astron. Soc. Australia, 22,118

James, B., Koposov, S., Stark, D.P., Belokurov, V., Pettini, M., Olszewski, E.W., 2015, MNRAS, 448, 2687 (J15)

James, B., Koposov, S., Stark, D.P., Belokurov, V., Pettini, M., Olszewski, E.W., McQuinn, K.B.W., 2016, MNRAS, in press (arXiv:1611.05888)

Hunter, D.A., Elmegreen, B.G., 2006, ApJS, 162, 49

Koda, J., Yagi, M., Yamanoi, H., \& Komiyama, Y. 2015, ApJ, 807, L2

Koposov, S.E., Belokurov, V., Torrealba, G., Evans, N.W., 2015, ApJ, 805, 130

Kormendy, J., Freeman, K.C., 2016, ApJ, 817, 84

Laher, R.R., Gorjian, V., Rebull, L.M., et al., 2012, PASP, 124, 737

Lange, R., Moffett, A., Driver, S., et al., 2016, MNRAS, 462, 1470

Lee, H., Grebel, E.K., Hodge, P.W., 2003, A\&A, 401, 141

Liske, Lemon, D.J., Driver, S.P., Cross, N.J.G., Couch, W.J., 2003, MNRAS, 344, 307

Liske, Baldry, I.K., Driver, S.P., et al. 2015, MNRAS, 452, 2087

Maddox, S.J., Sutherland, W.J., Efstathiou, G., Loveday, G., 1990, MNRAS, 243, 692

Marino, R. A., Rosales-Ortega, F. F., Sánchez, S. F., et al., 2013, A\&A, 559, 114

Martínez-Delgado, D., Laskër, R., Sharina, M., et al., 2016, AJ, 151,96

Mayer, L., Kazantzidis, S., Mastropietro, C., Wadsley, J., 2007, Nature, 445, 738

Majewski, S.R., Skrutskie, M.F., Weinberg, M.D., Ostheimer, J.C., 2003, ApJ, 599, 1082

McClure-Griffiths, N. M. et al., 2009, ApJS, 181, 398

McConnachie, A.W., 2012, AJ, 144, 4
McQuinn, K.D.W., Skillman, E.D., Berg, D.A., et al., 2013, AJ, 146,145

Mihos, J.C., Durrell, P.R., Ferrarese, L., et al., 2015, ApJ, 809, L21

Moore, B., Ghigna, S., Governato, F., et al., 1999, ApJ, 524, L19

Muñoz, R.O., Eigenthaler, P., Puzia, T.H., et al., 2015, ApJ, 813, L15

Niederste-Ostholt, M., Belokurov, V., Evans, N.W., Peñarrubia, J., 2010, ApJ, 712, 516

Osterbrock, D. E., \& Ferland, G. J. 2006, Astrophysics of gaseous nebulae and active galactic nuclei, 2nd. ed. by D.E. Osterbrock and G.J. Ferland. Sausalito, CA: University Science Books, 2006,

Pagel, B.E.J., Edmunds, M.G., Fosbury, R.A., Webster, B.L., 1978, MNRAS, 184, 569

Percival, S.M., Salaris, M., Cassisi, S., Pietrinferni, A., 2009, ApJ, 690, 426

Pettini, M., \& Pagel, B. E. J. 2004, MNRAS, 348, L59

Pietrinferni, A., Cassisi, S., Salaris, M., Castelli, F., 2004, ApJ, 612,168

Pilyugin, L. S., \& Mattsson, L. 2011, MNRAS, 412, 1145

Pilyugin, L.S., Grebel, E.K., 2016, MNRAS, 457, 3678

Pogge, R. W., Atwood, B., Brewer, D. F., et al. 2010, Proc. SPIE, 7735,9

Ricotti, M., 2009, MNRAS, 392, L45

Roberts, S., Davies, J., Sabatini, S., et al., 2004, MNRAS, 352, 478

Roediger, J.C., Courteau, S., 2015, MNRAS, 452, 3209

Roman, J., Trujillo, I., 2016, MNRAS, in press (arXiv:1610.08980) (RT16)

Sand, D.J., Crnojević, D., Bennet, P., et al., 2015, ApJ, 806, 95

Saul, D. R., Peek, J. E. G., Grcevich, J., et al. 2012, ApJ, 758, 44

Sawala, T., Frenk, C.S., Fattahi, A., et al., 2016, MNRAS, 457, 1931

Schlafy, E.F., Finkbeiner, D.P., 2011, ApJ, 737, 103

Shanks T. et al., 2015, MNRAS, 451, 4238

Stetson, P.B., 2005, PASP, 117, 563

Tollerud, E.J., Geha, M.C., Grcevich, J., et al., 2016, ApJ, 827, 89

van der Burg, R.F.J., Muzzin, A., Hoekstra, H., 2016, A\&A, 590, 20

van Dokkum, P. G., Abraham, R., Merritt, A., et al. 2015a, ApJ, 798, L45

van Dokkum, P. G., Romanowsky, A.J., Abraham, R., et al., 2015b, ApJ, 804, L45 van Dokkum, P. G., Abraham, R., Merritt, A., et al. 2015a, ApJ, 798, L45

van Dokkum, P. G., Abraham, R., Brodie, J., et al., 2016, ApJ, $828, \mathrm{~L} 6$

Vincenzo, F., Belfiore, F., Maiolino, R., Matteucci, F., Ventura, P., 2016, MNRAS, 458, 3466

Yagi, M., Koda, J., Komiyama, Y., Tamanoi, H., 2016, ApJS, 225, 11

Zaritsky, D., 2017, MNRAS, 464, L110

This paper has been typeset from a $\mathrm{T}_{\mathrm{E}} \mathrm{X} / \mathrm{L}_{\mathrm{A}} \mathrm{T} \mathrm{X}$ file prepared by the author. 